\title{
Non-linear unsteady aerodynamic model for insect-like flapping wings in the hover. Part 2: implementation and validation
}

\author{
S A Ansari*, R Żbikowski, and K Knowles \\ Department of Aerospace, Power, and Sensors, Cranfield University, Defence Academy of the United Kingdom, \\ Shrivenham, UK
}

The manuscript was received on 4 October 2005 and was accepted after revision for publication on 3 January 2006.

DOI: 10.1243/09544100JAERO50

\begin{abstract}
The essence of this two-part paper is the analytical, aerodynamic modelling of insect-like flapping wings in the hover for micro-air-vehicle applications. A key feature of such flapping-wing flows is their unsteadiness and the formation of a leading-edge vortex in addition to the conventional wake shed from the trailing edge. What ensues is a complex interaction between the shed wakes, which, in part, determines the forces and moments on the wing. In an attempt to describe such a flow, two novel coupled, non-linear, wake integral equations were developed in the first part of the paper. The governing equations derived were exact, but did not have a closed analytical form. Solutions were, therefore, to be found by numerical methods and implemented in Fortran. This is the theme of the second part of the paper. The problem is implemented by means of vortex methods, whereby discrete point vortices are used to represent the wing and its wake. A number of numerical experiments are run to determine the best values for numerical parameters. The calculation is performed using a time-marching algorithm and the evolution of the wakes is tracked. In this way, both flow field and force data are generated. The model is then validated against existing experimental data and very good agreement is found both in terms of flow field representation and force prediction. The temporal accuracy of the simulations is also noteworthy, implying that the underlying flow features are well captured, especially the unsteadiness. The model also shows the similarity between two-dimensional and three-dimensional flows for insect-like flapping wings at low Reynolds numbers of the order of $R e \sim 200$.
\end{abstract}

Keywords: flapping wings, aerodynamic modelling, insect flight, micro-air vehicle design, vortex methods

\section{INTRODUCTION}

In the first part of this two-part paper [1] , a non-linear, unsteady quasi-three-dimensional analytical model for hovering flapping-wing flight was developed. In the formulation, the flow associated with insect-like flapping flight was decoupled into wake-free (quasisteady) and wake-induced (unsteady) components. Using the concept of radial chords and radial

\footnotetext{
${ }^{*}$ Corresponding author: Department of Aerospace, Power, and Sensors, Cranfield University, Defence Academy of the United Kingdom, Shrivenham SN6 8LA, UK. email: s.a.ansari@ cranfield.ac.uk
}

cross-planes [1, section 2.1.1], a blade-element-type method was used to extend the approach from two-dimensions to three-dimensions.

Because of the high angles of attack encountered in insect flight, separation is observed from both leading and trailing edges [2-4] in the form of a leading-edge vortex (LEV) and the usual trailing-edge wake. These wakes, together with the unsteady motion of the wing, determine the forces and moments on the wing. By enforcing the Kutta-Joukowski condition at the wake-inception points and by observing Kelvin's law that the total circulation in a control volume enclosing the wing and its wake is constant, the flow equations were solved in accordance with Laplace's equation. 
As a result, two non-linear wake integral equations were derived that, between them, described the flow in its entirety. Although these equations are exact, they do not have a closed analytical form and must be solved by numerical methods. Vortex methods are used to implement this solution and study the validity of the model. This is the aim of this part of the paper.

This paper is organized as follows. In section 2, a brief review of vortex methods shows the basic elements of the numerical method that are used to solve the time-dependent problem. This is followed by a section dedicated to the numerical implementation of the flapping-wing model (section 3). The conversion of the relevant equations to forms amenable to numerical calculation is discussed together with a description of the calculation procedure. A number of factors pertinent to the numerical procedures are also addressed. The section concludes with a discourse on numerical experiments that were run to establish the values of various parameters. In section 4 , the validation of the current model is discussed in detail. The first part of this section considers comparison of flow-visualization from some existing experiments with results from the current model. In the next part of this section, force data are compared with experimental values from the literature. A description of the flow kinematics is also presented and the section concludes with the identification of some limitations of this model. Finally, in section 5, conclusions for the current paper as well as for the whole study are presented.

In what follows, frequent reference is made to the work presented in the first part of this paper [1] and is referred to as Part 1.

\subsection{Vortex methods}

All real flows feature some form of rotational motion developed in regions adjacent to flow surfaces or in the downstream wake of bodies. Another feature of real flows is separation, usually originating at sharp corners or produced as a result of adverse pressure gradients. Vortex methods attempt to model these flows by discretization of the distributed vorticity or separated shear layers by a finite number of small discrete vortex elements and this is the approach used to solve the non-linear integral equations developed in Part 1 [1].

Historically, Rosenhead [5] was probably the first to study the unsteady motion of two-dimensional vortex sheets. In his method, a vortex sheet is replaced by a (small) finite number of discrete point vortices that are 'free' (they cannot sustain Kutta-Joukowski forces) and, hence, move at the local Kirchhoff velocity [6]. The convection of the vortices is governed by the Rott-Birkhoff non-linear integro-differential equation $[\mathbf{7}, \mathbf{8}]$, which is essentially an extension of the Biot-Savart law and states that

$$
q(\zeta)=-\frac{\imath}{2 \pi} \oint_{C} \frac{\mathrm{d} \Gamma^{\prime}}{\zeta(\Gamma, t)-\zeta\left(\Gamma^{\prime}, t\right)}+\left(U_{\mathrm{E}}-\imath V_{\mathrm{E}}\right)
$$

where $\left(U_{\mathrm{E}}, V_{\mathrm{E}}\right)$ are the components of the external irrotational velocity field evaluated at $\zeta$, circulation $\Gamma$ is the Lagrangian variable, and ' (the 'prime' symbol) refers to all vortices other than the one in question.

A number of vortex convection schemes are found in the literature. The most common is the simple forward Euler scheme. This is a first-order scheme and is used here in a modified form to include sub-time-steps for increased accuracy, thus

$$
\zeta(t+\delta t)=\zeta(t)+n \sum_{i=1}^{n} q_{i}(t) \frac{\delta t}{n}
$$

where $\delta t$ represents the duration of each time-step and $n$ is the number of substeps per time-step and where the convection velocity $q_{i}$ is evaluated at each sub-time-step.

As the distance between a pair of vortices diminishes, the error of the Euler scheme increases, because induced velocities become very large (see equations (1) and (2)). It has, therefore, become commonplace to use 'desingularization' schemes to remove the infinite velocities at vortex centres. Probably the most common approximation is to use the Lamb-Oseen vortex, which is based on an exact Navier-Stokes solution of the one-dimensional laminar flow around a single viscous vortex in an unbounded incompressible domain $[\mathbf{9 , 1 0}]$. In the method used here, the model proposed by Vatistas et al. [1] is used, which gives a very good approximation to the Lamb-Oseen vortex (Vatistas et al. actually gave a more generic form. The version quoted above is of order 2 and shows good agreement with the Lamb-Oseen vortex.), thus

$$
v_{\theta}(r)=\frac{\Gamma}{2 \pi} \frac{r}{\sqrt{r_{\mathrm{c}}^{4}+r^{4}}}
$$

where $v_{\theta}(r)$ is the circumferential velocity at a distance $r$ from the vortex centre and $r_{\mathrm{c}}$ is the vortexcore radius.

Another way of limiting the effects of the unrealistically high induced velocities on vortices in close proximity is to amalgamate them, i.e. merge two or more vortices into one representative vortex. This method is used here because of its added benefit of reducing computation time (CPU flops). The usual rules of vortex amalgamation are observed: circulation and impulse of circulation are preserved 
during amalgamation [12-14]. Two vortices are merged if they are of like-sign and when the distance between their vortex centres falls below a certain value. In this case, the criterion proposed by Spallart [12] was used; vortex merging began when

$$
\left|\frac{\Gamma_{i} \Gamma_{j}}{\Gamma_{i}+\Gamma_{j}}\right| \delta_{i j}<\varepsilon
$$

where $\Gamma_{i}$ and $\Gamma_{j}$ are the circulations of the vortices to be merged, $\delta_{i j}$ is their separation and $\varepsilon$ is a small number.

\section{IMPLEMENTATION}

The current approach is formulated as an initialvalue problem, in that, the initial conditions are known and the flow is solved for all subsequent times using a time-marching algorithm.

In the unsteady motion of an aerofoil, the kinematic boundary condition induces velocities over the aerofoil surface. These upset the Kutta-Joukowski condition that the trailing edge must be a stagnation point. A similar condition holds for the leading edge where separation is also observed in insect-like flapping wings. The Kutta-Joukowski condition is, therefore, enforced by the 'creation' of bound circulation.

In the current formulation, a pair of vortices is shed at every time-step, one each from the leading and trailing edges in accordance with Kelvin's law and to satisfy the Kutta-Joukowski conditions there. Over the duration of the time-step, these 'free' vortices convect with the local Kirchhoff velocity, so that at the next time-step, the Kutta-Joukowski condition is disturbed once again. In addition, the kinematics of the wing and the associated boundary condition may have changed so that the velocity distribution on the wing is altered further. The Kutta-Joukowski condition is restored by shedding another pair of vortices from the respective edges. The shed vortices are convected at the local velocity. This process repeats over time and wakes from both separation edges evolve. A spinoff of the method is that a time history of the evolution of the wake is automatically generated, which is useful in demonstrating the physics of the flow.

\subsection{Discretization of equations for numerical solution}

Some of the equations derived in Part 1 [1, section 3] particularly non-linear and do not have closed-form solutions. Therefore, they must be solved using numerical schemes. Before numerical methods can be applied, however, the equations (as necessary) must be converted to forms that are amenable to numerical computation.
The equations for quasi-steady (wake-free) circulation are straightforward and can be computed directly from their derived forms (Part 1 , equations (17) and (18)). The expression for wake-induced vorticity (Part 1, equation (26)) is a little more complex as it involves non-linear wake integrals. In its discretized form, it is given by

$$
\begin{aligned}
\left.\gamma_{1}(\theta, t)\right|_{\mathrm{wk}+\mathrm{lv}}= & -\frac{\Gamma_{0}(t)}{2 \pi R}-\frac{1}{2 \pi R} \\
& \times\left[\sum_{i=1}^{n_{\mathrm{wk}}} \mathrm{R}\left(\frac{\mathcal{Z}_{\mathrm{wk}}+R \mathrm{e}^{\imath \theta}}{\mathcal{Z}_{\mathrm{wk}}-\operatorname{Re}^{\imath \theta}}\right) \gamma_{\mathrm{wk}} \mathrm{d} \mathcal{Z}_{\mathrm{wk}}\right. \\
& \left.+\sum_{i=1}^{n_{\mathrm{lv}}} \mathrm{R}\left(\frac{\mathcal{Z}_{\mathrm{lv}}+R \mathrm{R}^{\imath \theta}}{\mathcal{Z}_{\mathrm{lv}}-\operatorname{Re}^{i \theta}}\right) \gamma_{\mathrm{lv}} \mathrm{d} \mathcal{Z}_{\mathrm{lv}}\right]
\end{aligned}
$$

where the subscripts wk and lv refer to the trailingedge wake and LEV, respectively, and $n$ refers to the number of shed vortices. Before equation (5) can be evaluated, however, all circulations $\delta \Gamma_{\mathrm{wk}_{i}}$ and $\delta \Gamma_{\mathrm{lv}_{i}}$ must be known. This is true for all circulations except for the latest shed vortices $\delta \Gamma_{\mathrm{wk}_{n}}$ and $\delta \Gamma_{\mathrm{lv}_{n}}$. The latter are computed by solving the two wake integral equations derived in Part 1 (Part 1, equations (22) and (25)). Although these equations involve non-linear integrals, upon discretization as in equation (5), they reduce to two linear simultaneous equations of the form $A x+B y=C$, which can be solved together for the circulations of the latest shed vortices $\delta \Gamma_{\mathrm{wk}_{n}}$ and $\delta \Gamma_{\mathrm{lv}_{n}}$ (full details in reference [15, section 5.1.2]). Actually, two 'unknowns', the positions of the latest shed vortices $\left(\mathcal{Z}_{\mathrm{wk}_{n}}\right.$ and $\left.\mathcal{Z}_{\mathrm{lv}_{n}}\right)$, still remain and these are estimated from the movement of the shedding edge and the position of the previous shed vortex (section 3.2.1).

\subsection{Calculation procedure}

The calculation process, which is shown in Fig. 1, was implemented by means of a Fortran routine (discussed subsequently). The basic procedure that is followed is outlined below.

1. The computation is started by loading the various variables, reading in simulation parameters, and initializing them to the desired values.

2. The wing planform to be solved for is divided into the required number of wing sections (blade elements), using the radial chord concept introduced in Part 1 (Part 1, section 2.1.1).

3. Because all wing sections are on the same wing, they share common global kinematics in terms of sweep parameters $(\phi, \dot{\phi})$, inclination parameters $(\theta, \dot{\theta})$, and pitch parameters $(\alpha, \dot{\alpha})$. These are computed next. 


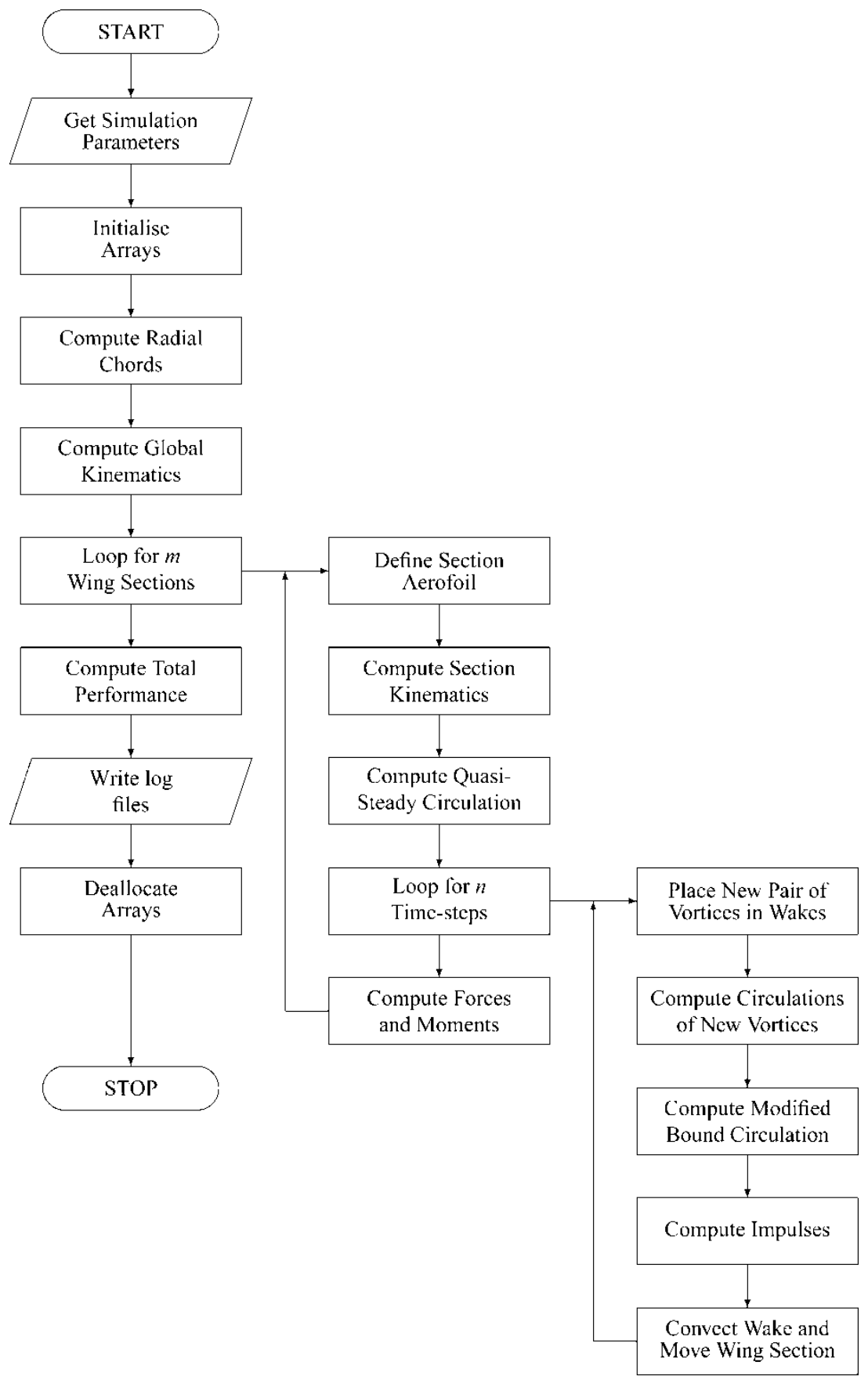

Fig. 1 Overview of calculation procedure

4. The program then descends into calculations for each spanwise section.

(a) First, the shape and coordinates of the aerofoil section are computed.

(b) Then, the specific kinematics for the wing section are deduced from the global kinematics computed earlier.

(c) With the information deduced thus far, the quasi-steady (wake-free) vorticity and circulation can computed.

These substeps are repeated for each wing section.
5. Now that all the basic and quasi-steady parameters for each wing section have been computed, the time-marching algorithm for the unsteady flapping-wing problem is invoked. All computations are carried out in the body-fixed coordinate system (in the $\zeta$ - or $\mathcal{Z}$-plane, as appropriate). At each time-step, the following processes are undertaken.

(a) The latest trailing-edge vortex and LEV are shed and positioned appropriately in the wake. 
(b) The circulations of these latest wake and LEV vortices are then computed by simultaneously solving the wake integral equations.

(c) The presence of the new vortices upsets the zero-through-flow boundary condition at the aerofoil surface, so this is corrected by adding additional bound vorticity. This is computed in this step using equation (5).

(d) The circulation and positions of all bound and shed vortices are used to compute the impulse and moment of impulse (for later force and moment calculation) in the translating aerofoil-inertia system.

(e) Now that all computations for the current time-step have been completed and all necessary variables determined, the entire wake system is convected and re-oriented in preparation for the, subsequent time-step, accounting for any change in pitch angle $(\alpha)$ and wing location $(l, h)$.

These above substeps are performed for each wing section.

6. Once time marching has been completed, forces and moments can be computed from the impulses and moments of impulse, respectively, that were computed earlier. This is done for each wing section.

7. The performance of the entire wing is obtained by summing the contributions of the individual wing sections.

8. Finally, data and $\log$ files for the simulation are written to disk, all arrays are de-allocated to free-up memory, and the simulation is terminated.

\subsubsection{Some aspects of the calculation procedure}

A number of aspects of the calculation need further detailing.

2.2.1.1 Forces and moments. Forces and moments are computed in the translating aerofoil-inertia system ( $\hat{\zeta}$-system in Part 1, Fig. 6) that moves with the aerofoil and remains horizontal throughout and in which the origin coincides with the wing pitch axis. Lift $\mathcal{L}$ is defined as the vertical force opposing gravity, acting in the direction of the positive $\hat{\eta}$-axis. Thrust $\mathcal{T}$ is defined as the horizontal force perpendicular to lift, but always acting in the direction of motion of the wing (along the $\hat{\xi}$-axis) and drag $\mathcal{D}$ is the net horizontal force (parallel to thrust, but always acting in the direction of the positive $\hat{\xi}$-axis).

2.2.1.2 Aerofoil discretization. The aerofoil shape in the physical $(\zeta)$ plane is derived from a circle in the $\mathcal{Z}$-plane using a Joukowski-type transformation
(Part 1, equation (1)). The aerofoil coordinates are equally spaced on the circle $\mathcal{Z}=R^{\imath}{ }^{\imath \theta}$ in the circle plane. The advantage of this is that, upon transformation into the physical plane, the resulting aerofoil has much tighter spacing in the leading- and trailing-edge regions than elsewhere. This is useful for accurately enforcing the Kutta-Joukowski condition at the two flow-separation edges.

2.2.1.3 Kinematics. The sweep angle at any point is defined by the angular displacement $\phi$ from the mean position. $\phi$ is generally positive at the start of the downstroke and negative at the end of it (Fig. 2). The reverse is true for the upstroke. The time rate of change of sweep is defined by the angular velocity $\dot{\phi}$ and the stroke amplitude is denoted by $\Phi$. In a similar manner, the inclination angle above the stroke plane is defined by $\theta$ and the time rate of change of inclination by the angular velocity $\dot{\theta}$ (Fig. 2).

Using the radial cross-plane analogy (Part 1, section 2.1.1), sweep and inclination are converted into lunge $(l, \dot{l})$ and heave $(h, \dot{h})$, respectively, where the (') refers to time rate-of-change. Pitch (or pitch rate) is defined as the angular displacement (or velocity) about the common pitch axis and is the same for all wing sections.

2.2.1.4 Vortex placement. Traditionally, the latest shed vortex is positioned along the path of the shedding edge (leading or trailing edge) since the last time-step [16, section 13.8.2]. The approach used here is similar, but the latest vortex is placed instead at one-third of the distance from the shedding edge to the previous vortex (equation (6)) (Fig. 3).

The use of the one-third distance can be explained as follows. The discrete point vortices that are shed are an approximation to the vortex sheet emanating from the shedding (leading or trailing) edge. Therefore, each shed vortex actually represents a vortex element of length, say $\delta \zeta$, and is placed approximately in the middle of this element. In this way, the centre of the latest shed vortex is $(1 / 2) \delta \zeta$ distant from the shedding edge (Fig. 3(a)).

At the next time-step, the last vortex has convected with the flow and the aerofoil has moved some distance. Assuming that the time-step is small enough, the new vortex element is of similar length to the previous one $(\approx \delta \zeta)$ and, as before, the new discrete vortex is positioned at its centre (Fig. 3(b)). The distance from the previous vortex to the shedding edge along the vortex sheet is then $\approx(3 / 2) \delta \zeta$, whereas the new vortex is $\approx(1 / 2) \delta \zeta$ from the same edge, i.e., the new vortex is located at about one-third of the distance along the vortex sheet to the previous shed vortex from the shedding edge (Fig. 3(b)). 


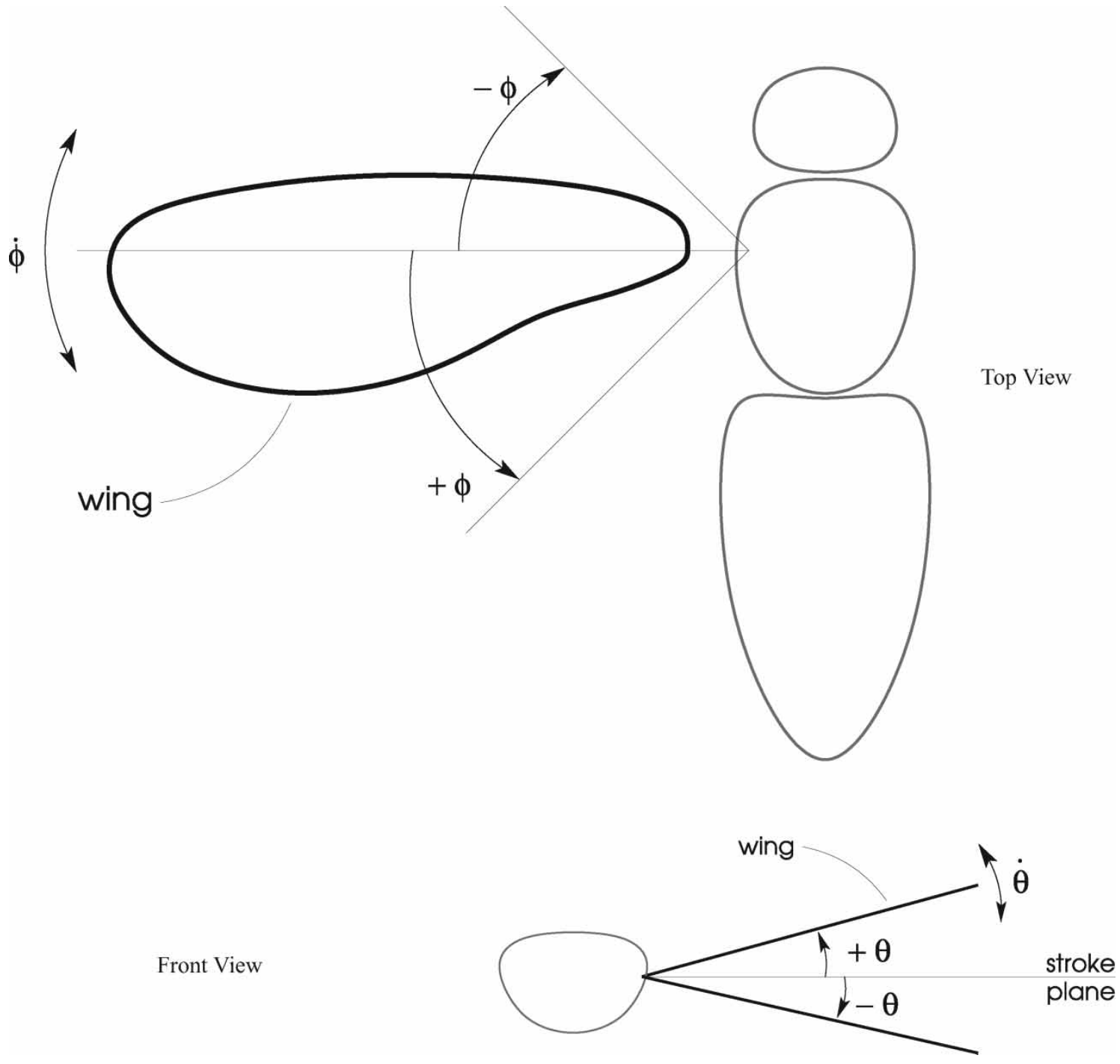

Fig. 2 Definition of sweep (top) and inclination (bottom) parameters. Only port wing is shown

The location of the new vortex is then approximated by drawing a vector from the shedding edge to the previous shed vortex and taking the position at one-third this distance, that is

$$
\zeta_{t}=\zeta_{\text {edge }}+\frac{1}{3}\left(\zeta_{\text {edge }}-\zeta_{t-1}\right)
$$

The advantage of the current approach over the traditional one is that account is taken not only of the wing motion since the last time-step but also of the convection of the previous shed vortex, giving overall a more accurate depiction of the flow.

As regards the first shed vortex, its position is determined from the local velocity at the shedding edge. If this complex velocity is $q$ and time-step size is $\delta t$, then the first vortex is placed at

$$
\zeta_{t}=\frac{1}{2} \bar{q} \delta t
$$

where $\bar{q}$ refers to the complex conjugate of $q$. As the solution progresses, the accuracy of this initial placement is of negligible consequence (provided timestep size is reasonably small).

2.2.1.5 Wake convection. At the end of each timestep, the whole problem is converted to the inertial coordinate system ( $\tilde{\zeta}$-system in Part 1, Fig. 6) and the wakes from each of the leading and trailing edges are convected using a discretized form of the Rott-Birkhoff equation (equation (1)). During stroke reversals, the severity of the wing motions warrants the need for finer resolution, and more sub-time-steps are used (section 4).

2.2.1.6 Sub-time-steps. The sub-time-stepping scheme utilized in the current work is based on equation (2) with $n$ set to 1,2 , or 4 , depending on the severity of the kinematics. A measure defined by the ratio of the normal kinematic velocity of the 


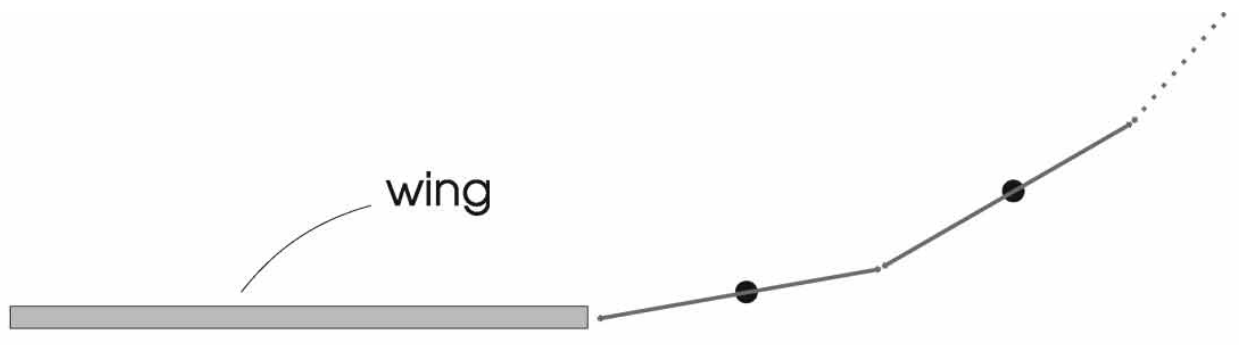

(a) Previous time-step

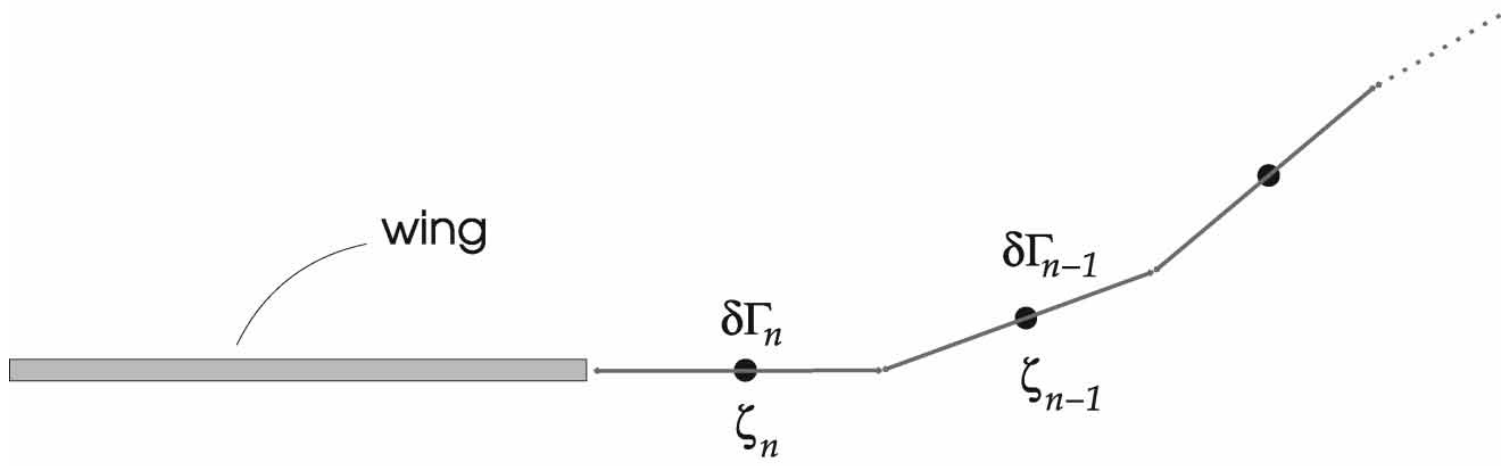

(b) Current time-step

Fig. 3 Vortex placement (wake deformations are exaggerated for clarity)

trailing edge to the number of chord lengths it travels in a time-step is used to set the value of $n$. By defining the ratio in this way, the number of sub-time-steps may be different for each wing section, depending on both chord length and local velocity.

2.2.1.7 FORTRAN code. The aforementioned computational schemes were implemented by means of a Fortran program. The choice of using Fortran as the programming language was mainly due to its intrinsic capability of handling complex numbers, which made for easy conversion of the complex-variablebased analysis in the current work into computable code. All computations were performed in double precision because of the extensive use of difference formulae in the computation of forces and moments (Part 1, section 3.2).

The size of the compiled program was quite small (of the order of 2.0 MB). Memory requirements, however, were determined at runtime, depending on the number of wing sections and time-steps. Typically, $10 \mathrm{MB}$ was required for 16000 section-time-steps (e.g. eight wing sections with 2000 time-steps each). The program was compiled using the Intel Fortran Compiler v. 8.0 on a Linux workstation running RedHat 9.0. The program was also run on the same machine, which was a single-processor Dell Precision 360 workstation with a $2.66 \mathrm{GHz}$ Intel Pentium 4 processor. A typical run with 6400 section-time-steps took $\sim 10 \mathrm{~min}$ to complete.
The bottleneck for the program was the convection subroutine, where approximately $4 n^{2}$ velocity computations were made at each time-step (where $n$ is the current time-step). Simulation times, however, scaled roughly as $n^{2.5}$ owing to the different number of sub-time-steps used by each wing section. Computation time also depended on the number of stroke reversals in the simulation (when more sub-time-steps were required) and also on whether vortex amalgamation was employed.

\subsubsection{Numerical modifications}

Owing to the nature of the numerical implementations and the limitations of the inviscid potential method on which they were based, situations arose where the behaviour of the wakes gave rise to high fluctuations in the associated forces and moments (owing to unrealistically large wake deformations). In reality, such variations are limited or dampened by viscosity. Therefore, it was necessary to introduce such limitations artificially as necessary.

2.2.2.1 Kutta-Joukowski condition relaxation. During the more extreme phases of insect-like flapping flight (such as stroke reversals), it was found that the newly shed vortices acquired circulations that could be as much as three orders of magnitude higher than the previous shed vortex, all in an effort to satisfy the Kutta-Joukowski condition at the 
vortex-shedding edge. In such cases, the subsequent vortex that was shed was also of similarly large strength but of opposite sense to ensure stagnation at the shedding edge, and the process continued for a number of time-steps. The situation occurred more commonly during wake re-entry when the wing's leading and/or trailing edge passed in close proximity to previously shed vortices.

It was, therefore, necessary to curb the circulation strengths of the shed vortices in such situations, thereby temporarily not satisfying the KuttaJoukowski condition (Crighton [17] considered the validity of the Kutta-Joukowski condition in unsteady flows. In practice, the condition appears to hold [18], especially for helicopter aerodynamics where the kinematics are not too severe [19].) for that particular time-step. In reality, viscosity is likely to introduce similar limitations. Indeed, on the basis of the assumption that the mean velocity of flow from the trailing edge is finite and non-zero, Giesing [20] was able to infer that the vortex sheet was shed parallel to either the upper or the lower surface of the trailing edge, depending on the direction of shed vorticity. Maskell [21] later came to the same conclusion. Mook and Dong [22] applied the Giesing-Maskell model and showed that the surface of separation could be deduced from the rate of change of bound circulation $\mathrm{d} \Gamma / \mathrm{d} t$. When $\mathrm{d} \Gamma / \mathrm{d} t>0$, the streamline along the lower surface leaves the aerofoil smoothly. Conversely, when $\mathrm{d} \Gamma / \mathrm{d} t<0$, the flow relative to the aerofoil comes smoothly off the upper surface. In the steady-state case of $\mathrm{d} \Gamma / \mathrm{d} t=0$, the stagnation streamline bisects the trailing-edge angle. Later experiments by Poling and Telionis $[\mathbf{2 3}, \mathbf{2 4}]$ corroborated these results.

During stroke reversals, the velocity vector of the flow at the aerofoil-trailing edge, for example, is closer to perpendicular to the aerofoil surface than it is parallel to it. In such cases, the smooth-flow Kutta-Joukowski condition is likely to be temporarily relaxed. Circulation strengths of shed vortices, therefore, were curbed by imposing a circulation limit. A recent computational fluid dynamics (CFD) study by Sun and Boyd [25] has shown that for a flat plate at a Reynolds number $R e=135$, the slip velocity on the aerofoil surface, especially near the leading and trailing edges was significant. Therefore, the concept of relaxing the Kutta-Joukowski condition (as proposed here) is not unrealistic in these flow regimes.

\subsubsection{Smoothing. Another artefact of the} numerical simulations was the appearance of spikes (both positive and negative) in the force and moment data, each time the wing entered its own wake and, especially, during stroke reversals. This is an artefact of the method used for computing forces and moments (Kelvin's impulse method). As a wing traverses through a wake, the free vortices part to either side of it, so that some vortex positions that were in 'positive' locations with respect to the translational aerofoil-inertia axis in one time-step suddenly acquire 'negative' positions, and vice versa. This leads to large changes in impulses and moments of impulse and, hence, artificial spikes in force and moment data (forces and moments are computed as the time rate of change of impulse and moment of impulse respectively (Part 1, section 3.2).

Such spikes increased as the number of flapping cycles increased owing to the increasing number of encounters with previously shed wakes. It was, therefore, necessary to remove such spikes and this was achieved by means of a simple least-squares smoothing technique.

\subsection{Numerical experiments}

The analytical approach used in the current work relies in its implementation on the use of numerical techniques. Hence, it is inevitable that some dependence on numerical parameters exists. A study was, therefore, conducted to describe these characteristics of numerical solutions with a view to determine the best values for the numerical parameters. This was achieved by running a number of numerical experiments.

Two different wing shapes were used for this series of investigations - Dickinson's Robofly wing (Fig. 4(a)) and a wing shape derived from four elliptical arcs (similar to that of a hoverfly) see (Fig. 4(b)) and referred to as generic from hereon. The reason for using two wing shapes was to remove any dependence of the numerical parameters on wing geometry. The kinematics were provided by Dickinson (private communication) from his Robofly experiments $[4,26]$, and are henceforth referred to as Dickinson's data (Fig. 8).

\subsubsection{Effect of number of wing sections}

The wing was radially divided into a number of sections $n_{\text {sec }}$ and the overall effect achieved by summing the individual contributions. A high number of wing sections gives better resolution in terms of force and moment data but comes at the cost of computing time and power. Hence, it was necessary to determine the minimum number of wing sections that could reasonably reproduce the results at high resolution. A study on this effect showed that mean forces converged for $n_{\mathrm{sec}} \geqslant 20$, implying that this value of $n_{\text {sec }}$ would yield the best results within the accuracy of the model. 


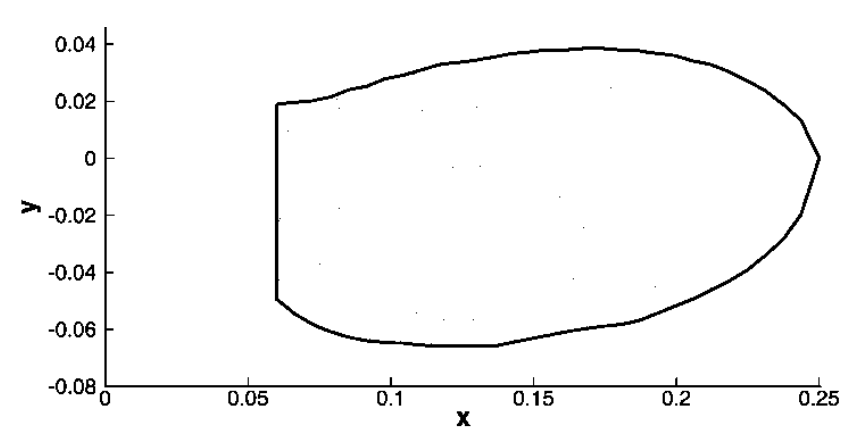

(a) Robofly wing

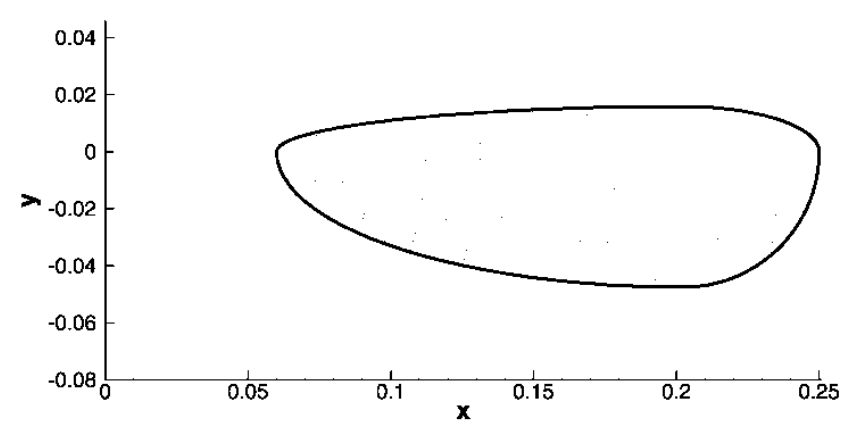

(b) Generic wing

Fig. 4 Wing planforms used for numerical experiments

\subsubsection{Effect of aerofoil discretization}

Aerofoil discretization $n_{\text {foil }}$ refers to the number of elements that the aerofoil is divided into. Apart from enforcing the zero-through-flow boundary condition more rigorously, higher aerofoil discretization reduces the chances of free vortices accidentally penetrating the wing during the convection phases of the computation. However, the investigation into its effect showed that aerofoil discretization had little influence on the overall result and a value of $n_{\text {foil }}=50$ was chosen conservatively.

\subsubsection{Effect of time-step size}

Although time-step size is bound to influence the solution, it is not a universal parameter in that it shows different trends for the same wing shape at different flapping frequencies. A faster wing moves further in the same time than a slower wing, and therefore, demands higher time resolution to yield accurate results. A different parameter was, therefore, defined, which would circumvent the limitation of the time-step: the mean angle swept by the wing per time-step.

For the cases investigated, the simulations were run for one cycle (downstroke and upstroke), which swept two arcs of $180^{\circ}$ each as per Dickinson's kinematics. The trend from this study showed that force data generally got less peaky with decreasing time-step size (or mean angle per time-step). Careful consideration of the trend showed that a time-step size of $0.01 \mathrm{~s}$ (mean angle per time-step of $0.6^{\circ}$ ) gave the best compromise between large time-step size and convergent results, and was chosen for all subsequent simulations.

\subsubsection{Effect of vortex-core size}

In the current implementation, the Vatistas vortex has been used to desingularize the potential vortex (equation (3)) so that each vortex exhibits a solidbody rotation near its centre and a constant vortexcore size was used throughout the simulation. In this study, vortex-core size appeared to have negligible effect on the overall performance except for very large values where the force data began to diverge from the 'norm' due to insufficient roll-up.

This argument would imply benefit in using small vortex cores. However, as vortex-core size reduces, the potential vortex limit is approached and the induced velocities near its centre become unrealistically high. The result is that vortices in close proximity begin to orbit each other, giving rise to jagged vortex sheets. Therefore, an intermediate value of vortex-core size was chosen for subsequent computations as the best compromise for smooth vortex sheets and sufficient roll-up. This value of vortexcore radius corresponded to approximately 2.5-5.0 per cent of the wing section chord.

\subsubsection{Effect of vortex amalgamation}

The benefit in computing time due to vortex amalgamation comes at the cost of accuracy of the calculations and of flow representation. It was, therefore, necessary to determine the limit to which this process could be exploited before adverse effects on the accuracy of the calculation surfaced. This numerical experiment showed that savings in computation times only became significant for $\epsilon>$ $5 \times 10^{-7}$ (where $\epsilon$ is the amalgamation threshold) (equation (4)). As regards the effect on the accuracy of the computation (measured here in terms of deviation from the amalgamation-free results), this became noticeable only beyond $\epsilon>10^{-6}$. In view of this finding, a value of $\epsilon=10^{-6}$ was used for the amalgamation threshold $\epsilon$.

One aspect of vortex amalgamation that is not immediately obvious is the possibility of abnormally large convection of adjacent vortices. Vortices of like-sign merge to form stronger vortices. Therefore, it is possible for two groups of vortices of opposite senses in close proximity to merge into two large vortices of opposite signs (Fig. 5), which may then convect far away from the wing owing to mutual 


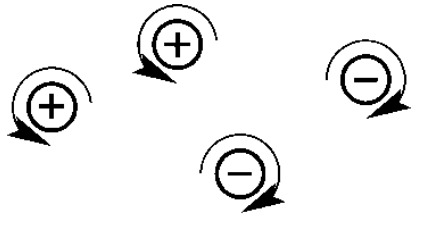

Bęfore

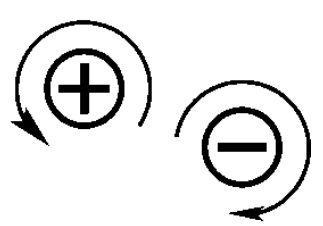

After

Fig. 5 Vortex amalgamation problems

convection. Therefore, vortex amalgamation must be implemented with caution.

\section{VALIDATION}

The model developed in the current work was tested and validated against experimental data for the assessment of its validity and limitations. Data from two sets of experiments were used for this model-verification process. The first data set was adapted from the experiment of Dickinson and Götz [27] for flow field comparison to assess the verity with which the current theoretical model captured the physics of the flow. Force data from Robofly experiments provided by Dickinson (private communications) served as the second data set for the validation of the lift and thrust predictions.

\subsection{Flow visualization comparison with Dickinson and Götz [27]}

The original experiment was conducted by Dickinson \& Götz [27] in a glass aquarium filled with a 54 per cent sucrose solution. A rectangular wing with $5 \mathrm{~cm}$ chord and $15 \mathrm{~cm}$ span was traversed in a straight line and at constant angle of attack through this solution between a pair of baffles to limit any three-dimensional tip flow. The wing was impulsively started, accelerated at $62.5 \mathrm{~cm} / \mathrm{s}^{2}$ to a constant speed of $10 \mathrm{~cm} / \mathrm{s}$, and then rapidly brought to rest after travelling $37.5 \mathrm{~cm}$ (7.5 chord lengths). Experiments were run for wing angle of attack ranging from $-9^{\circ}$ to $+90^{\circ}$ in steps of $4.5^{\circ}$. The Reynolds number for the experiment was 192 (based on chord).

The conditions for the Dickinson and Götz experiment were reproduced, and the flow equations were solved for using the current inviscid potential model. The wing was replaced by a thin, flat plate (The wing used in the Dickinson and Götz experiment was $1 \mathrm{~mm}$ thick giving it a thickness-to-chord ratio of 2 per cent making the thin-wing assumption a valid one.), and only the cases of large angles of attack $\left(\alpha \geqslant 22.5^{\circ}\right)$ were considered, where Dickinson and Götz had identified the definite presence of a LEV.
The kinematics for the experiment are shown in Fig. 6.

Dickinson and Götz presented flow-visualization photographs for the case of angle of attack of $45^{\circ}$. These are compared with the results from the current model in Fig. 7. Good agreement can be seen between the viscous experiment and the inviscid, theoretical prediction, despite the very low Reynolds number.

The flow-visualization photographs were for fixed instances in time ranging from one to four chord lengths of travel. After one chord length of travel, flow has begun to separate from both leading and trailing edges (Fig. 7(a)). Flow from the leading edge has rolled up very tightly because of the influence of the bound vorticity on the wing. The trailing-edge vortex has also rolled up albeit less than the LEV, being 'freer' than the latter. As the wing moves, roll-up continues in both vortices, but the LEV also grows significantly in size, lifting off slowly from the wing surface (Fig. 7(b)).

At this point, the original trailing-edge vortex has reached a near-constant size but continues to roll up. Owing to its growing size and strength, the LEV causes flow from the trailing edge to roll up into a second trailing-edge vortex that spills forward onto

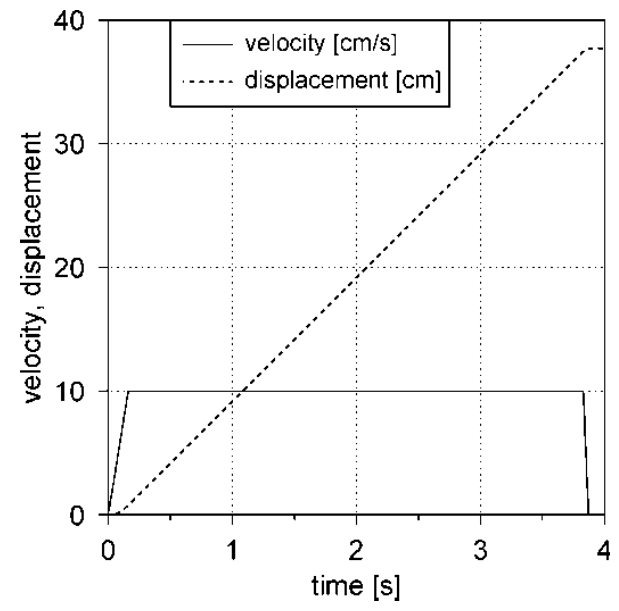

Fig. 6 Translational motion kinematics used by Dickinson and Götz [27] 

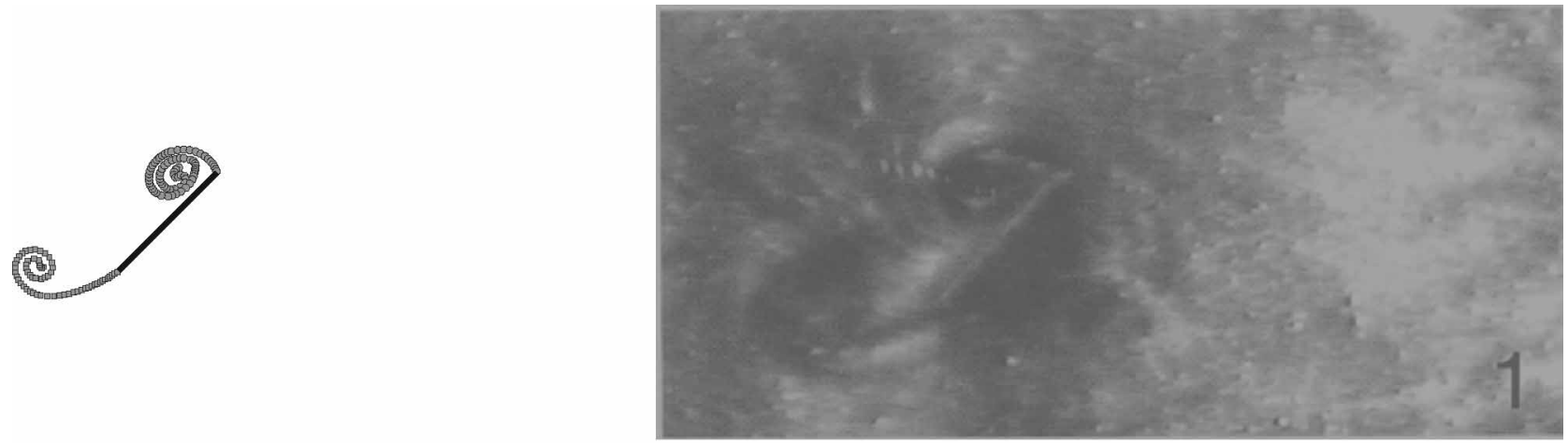

(a)
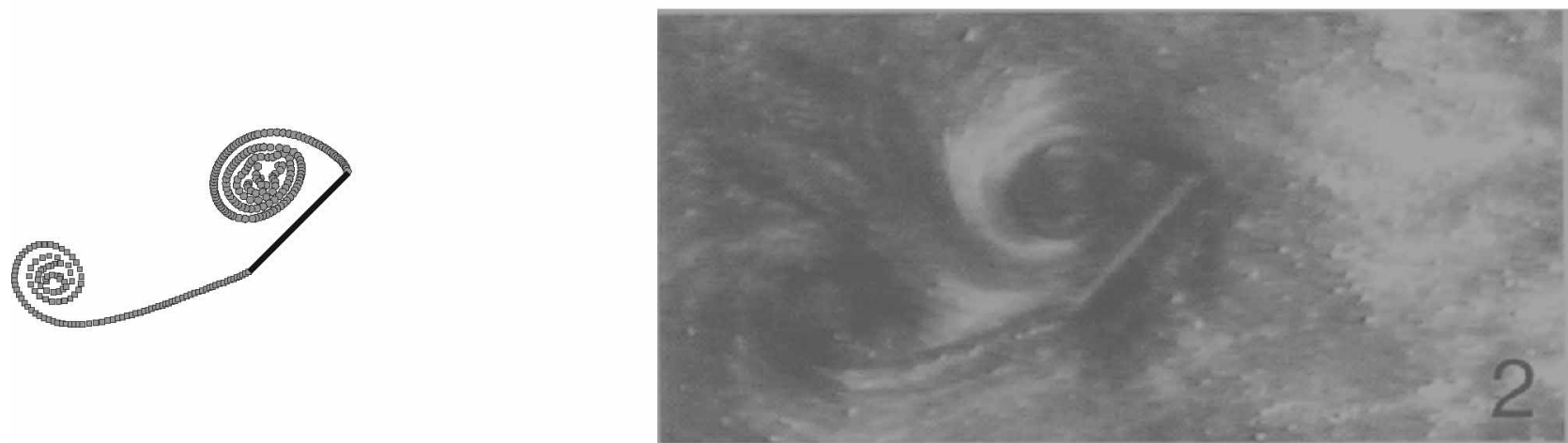

(b)

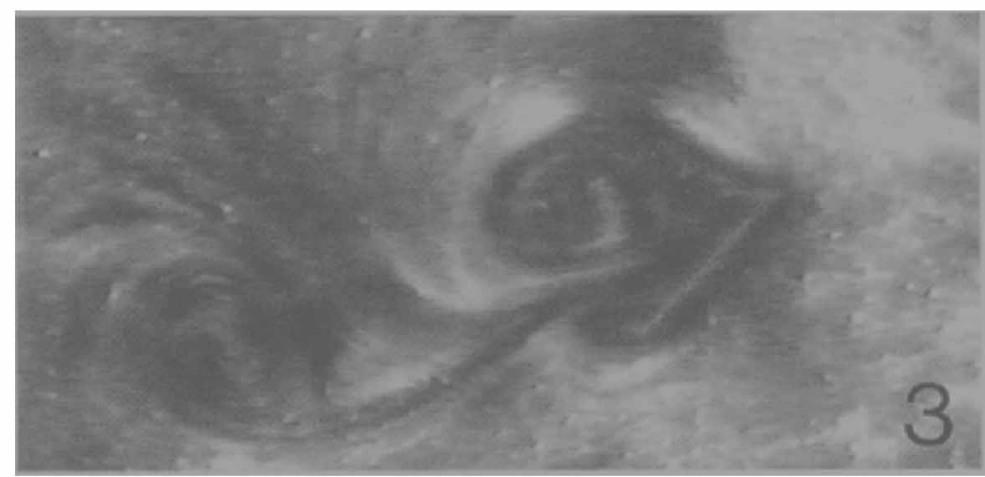

(c)

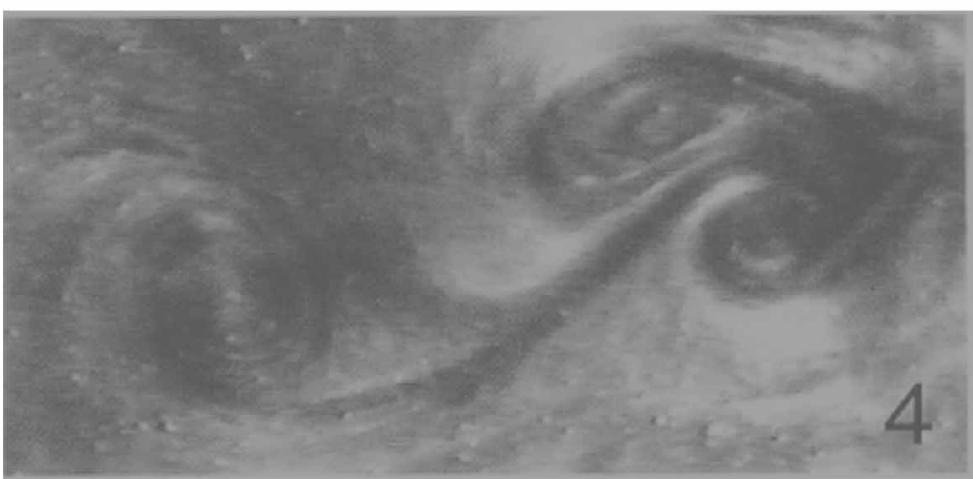

(d)

Fig. 7 Comparison of flow-visualization results from the current inviscid theoretical model (left) with the Dickinson and Götz [27] experiment at $R e=192$ (right). The numbers 1 to 4 refer to the number of chord lengths travelled since impulsive start 
the wing surface (Fig. 7(c)). This roll-up eventually leads to the distortion of the LEV and finally vortex breakdown occurs (Fig. 7(d)). The theoretical prediction even captures the triangular structure on the right-hand side of the rolled-up end of the distorted LEV after four chord lengths of travel (Fig. 7(d)).

Eventually, the LEV breaks away from the wing (data not shown) and a new one begins to form. As this second LEV begins interaction with the existing second trailing-edge vortex, the wing is rapidly brought to a halt. The result is the shedding of two stopping vortices, one each from the leading and trailing edges.

\subsection{Force comparison with Robofly experiments}

The comparison presented in this section is based on the data provided by Dickinson (private communication) from the setup that was used by Birch and Dickinson [4]. A scaled-up model of the wing of the fruit fly Drosophila dubbed the Robofly was used (Fig. 4(a)), in which the wing executed an insectlike flapping motion at a frequency of $\sim 0.17 \mathrm{~Hz}$ with the wing tip tracing out a flat figure-of-eight. The wing swept a semicircular $\operatorname{arc}\left(\Phi=180^{\circ}\right)$, this being possible, because only one wing was used (no mechanical interference from the 'other' wing). The experiment was conducted in a tank of mineral oil (of density $880 \mathrm{~kg} / \mathrm{m}^{3}$ ), which produced a Reynolds number of 160 (based on mean chord and mean tip speed). Data were provided for four complete cycles (or eight halfstrokes) starting from rest and executing the kinematics shown in Fig. 8. The wing sweeping velocity is constant for most of the halfstrokes, but reverses direction at each stroke reversal (Fig. 8(a)). Angle of attack also follows a similar routine - it remains at $45^{\circ}$ for most of the halfstrokes but goes through a $90^{\circ}$ rotation at stroke reversal (Fig. 8(b)). The pitching motion of the wing leads the sweeping motion by about 6 per cent of the flapping cycle (c.f. Figs 8(a) and (b)). Also, pitch rates are relative to the sweeping motion so that an increasing angle of attack before stroke reversal becomes a decreasing angle of attack once the subsequent halfstroke begins.

The computational simulations for the above experiment were run using the best combination of the parameters inferred from the numerical experiments described earlier. This benchmark simulation was one of the longest owing to the 48000 sectiontime-steps $(=20$ wing sections $\times 2400$ time-steps) with as many as four sub-time-steps per time-step. The results from the simulation are compared with the experimental data in Fig. 9.

The similarity between the experimental data and the results of the numerical simulation is immediately obvious. The pair of opposite spikes at
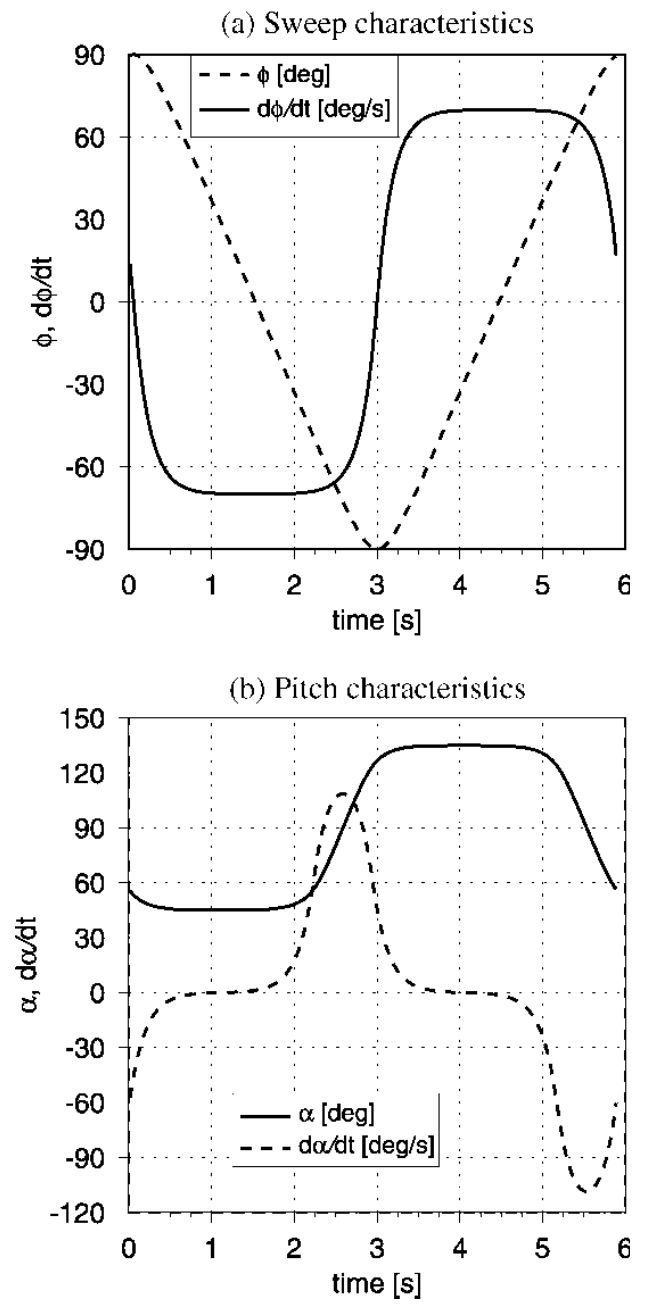

Fig. 8 Kinematics of Dickinson's Robofly. Because the wing swept a flat figure-of-eight, the inclination parameters $(\theta, \theta)$ were zero and are not shown

stroke reversals are particularly well captured by the theoretical model. They occur at the same points in time without any significant lag for both lift and thrust, thus accounting well for unsteadiness of the flow. Moreover, the magnitudes of the negative spikes for lift (and positive spikes for thrust) are consistent with the experimental data but the opposite spikes are overestimated. (Dickinson did not comment on the accuracy of the instrumentation used in his experiments.) Of the two, the force predictions for thrust are the better depicted.

These results are encouraging, because they show better agreement than the previously published comparisons, e.g. the CFD study by Sun and Tang [28] showed relatively poor comparison with the experiments of Dickinson et al. [29].

The spikes in lift (Fig. 9(a)) correspond to the latter phases of the halfstrokes when angle of attack increases rapidly (Fig. 8(b)) as stroke reversal occurs. Almost immediately afterwards, the wing 


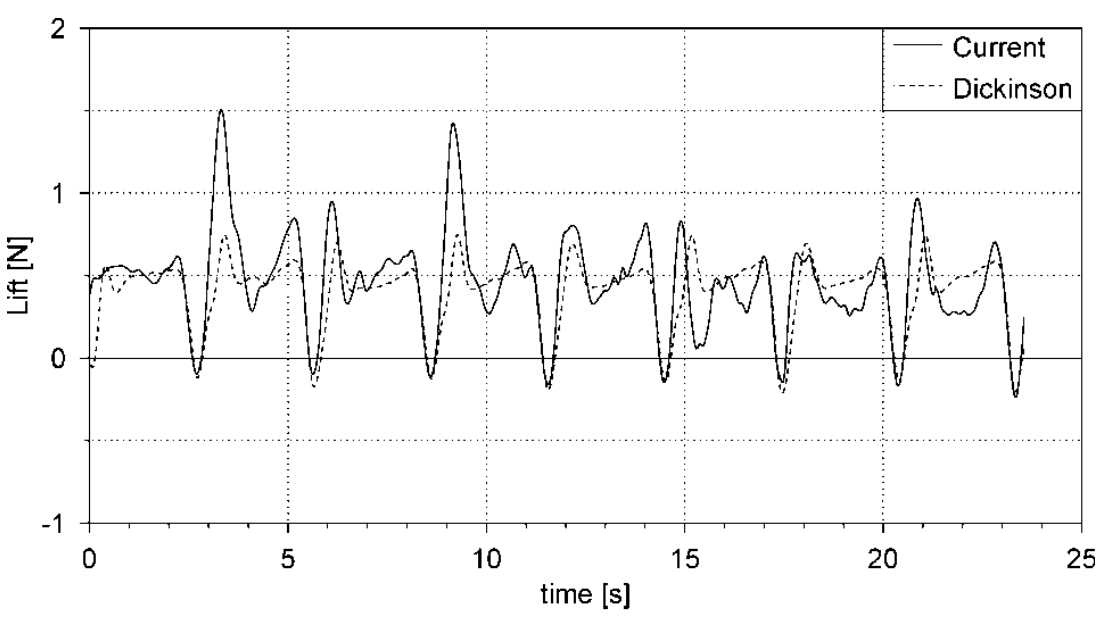

(a) Lift comparison

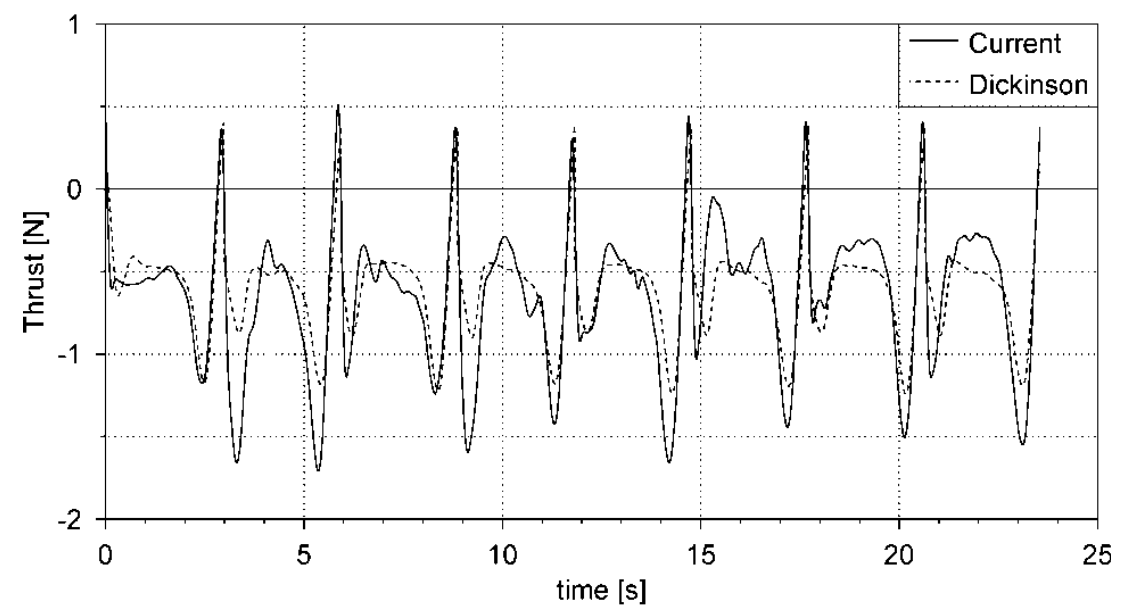

(b) Thrust comparison

Fig. 9 Comparison of force data for Robofly data from Dickinson (private communication) with results from the current theoretical simulation

translational velocity drops to zero, manifesting in a sharp decrease in lift. When wing motion begins in the opposite direction, the angle of attack is still relatively high and a second spike in lift is observed. This is, however, short-lived because the angle of attack drops rapidly as stroke reversal ends. As both lift and thrust derive from the same normal force, corresponding peaks and troughs are found in the thrust plot (Fig. 9(b)). A more detailed discussion of the flow physics is presented in section 4.2.1.

An estimate of the quality of the theoretical model can be inferred by comparing the mean values of lift $\mathcal{L}$ and thrust $\mathcal{T}$ (as defined in section 3.2.1.1). These are shown in Table 1. Although no allowance has been made to incorporate any three-dimensional tip effects directly, the comparison is promising. Another check is to compare the values for mean lift and thrust for the last cycle of the simulation where the forces are becoming reasonably periodic (also shown in Table 1). This comparison is consistent, whereas the inviscid model underestimates both lift and thrust.

Further substantiation is offered by the trend in drag $\mathcal{D}$ (the net horizontal force; section 3.2.1.1). This must be close to zero for steady hovering. From Dickinson's data, mean $\mathcal{D}$ for the last cycle is $0.0055 \mathrm{~N}$ which makes it 0.9 per cent of mean $\mathcal{T}$ for the same period. For the theoretical model, the respective values are $6.29 \times 10^{-4} \mathrm{~N}$ and a ratio of

Table 1 Comparison between experimental and theoretical values for mean lift and mean thrust

\begin{tabular}{lccllc}
\hline & \multicolumn{2}{c}{ Entire simulation } & & \multicolumn{2}{c}{ Last cycle } \\
\cline { 2 - 3 } \cline { 5 - 6 } & Lift (N) & Thrust (N) & & Lift (N) & Thrust (N) \\
\hline Dickinson & 0.4034 & -0.6011 & & 0.4081 & -0.6106 \\
Current & 0.4568 & -0.6445 & & 0.3805 & -0.5904 \\
Discrepancy (\%) & 13 & 7.2 & & 6.8 & 3.3 \\
\hline
\end{tabular}


about 0.1 per cent - an even better condition for hover. The inference to be drawn here is that the forces are becoming reasonably periodic after four cycles.

Another noteworthy feature is the synchrony between the experimental measurements of Dickinson and the theoretical predictions yielded by the current inviscid model. Irrespective of magnitude, the positions of all peaks are consistent with the experiment. The quality of this synchrony can be better appreciated by considering the power spectral densities of the force data obtained from their fast Fourier transform (Fig. 10). As with force data, the similarity between the experimental and theoretical values here is remarkable, and more so for thrust (Fig. 10(b)). This also shows that the smoothing scheme used (section 3.2.2) preserves the frequency content of the data.

The frequency content for Dickinson's data shows $0.3418 \mathrm{~Hz}$ as the dominant frequency, together with a number of harmonics. This is the blade-passing frequency, arising from there being two halfstrokes per cycle. The sources for the main peaks and troughs are the stroke reversals at the end of each halfstroke when vortices are shed and new ones form (discussed subsequently). Recalling that the flapping

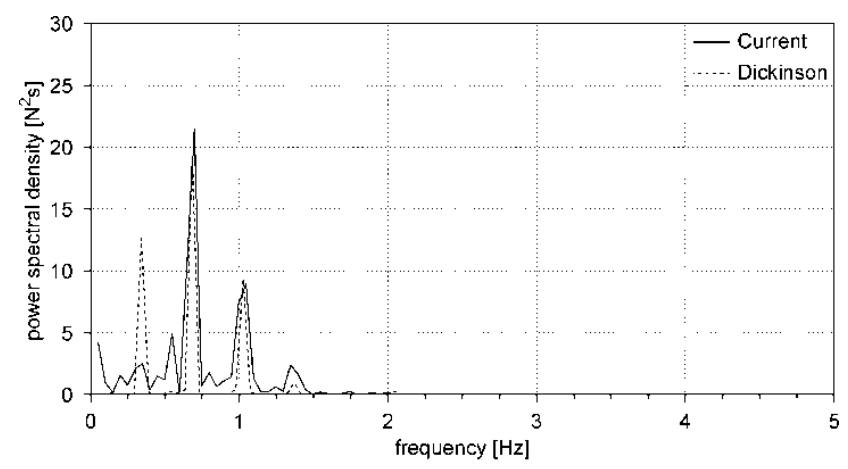

(a) Lift comparison

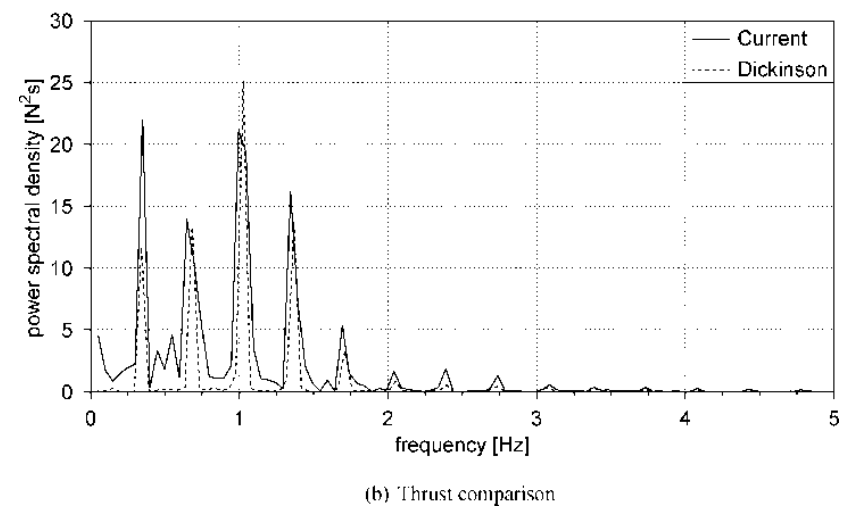

Fig. 10 Comparison of power spectral density of Robofly force data from Dickinson (private communication) with results from the current theoretical simulation frequency for the motion was $\sim 0.17 \mathrm{~Hz}$, the 'vortexshedding frequency' is twice that or $\sim 0.34 \mathrm{~Hz}$. In this model, the corresponding dominant frequency is found to be $0.3483 \mathrm{~Hz}$ - a very close match to experiment - together with similar harmonics. This frequency is not very clearly observed for the lift data, because the two large positive peaks in the second and fourth halfstrokes (Fig. 9(a)) dominate. However, higher harmonics are clearly visible (Fig. 10(a)).

The fact that frequency content for the experimental and theoretical data is so similar signifies that the underlying physical phenomena (e.g. vortex shedding) are captured well. Recalling that the quasi-three-dimensional model uses a simple blade-element-type method, it implies that the three-dimensional flow field associated with a flapping wing (at these Reynolds numbers) does not differ much from an aggregation of the flow field in each of its two-dimensional sections. This substantiates the use of the blade-element method.

The inference to be drawn from this discussion is that the aerodynamic model developed in the current works $[\mathbf{1}, \mathbf{3 0}]$ predicts with remarkable accuracy both the forces and flow-field associated with insect-like flapping wings. The temporal accuracy of the prediction implies that the unsteadiness in the flow is also well captured by the theoretical model. Further, for the Reynolds numbers in question, the threedimensional flow associated with such wings appears to be essentially equivalent to two-dimensional flow.

\subsubsection{Characteristics of the flow}

The trend observed for lift force $\mathcal{L}$ is that upon startup lift grows rapidly from zero (Fig. 9(a)). Thereafter, it remains more or less constant for most of the halfstroke. At the end of the halfstroke, there is a steep negative spike, which is immediately followed by a sharp positive spike. This trend then repeats in a similar fashion for all subsequent halfstrokes.

At startup, angle of attack is quite high but sweeping velocity is low (Figs 8(a) and (b)) so that its effect on lift is low. However, the newly formed LEV increases lift significantly, which is manifested as an initial spike (Fig. 9(a)). As the wing accelerates from rest, a trailing-edge vortex is also shed, whose primary effect is to limit the value of lift. The net result is to keep lift more or less constant during most of the halfstrokes (Fig. 9(a)).

At the end of the halfstroke, stroke reversal occurs - there is a simultaneous increase in angle of attack and a decrease in sweeping velocity (Figs 8(a) and (b)) with the net effect of reducing lift. Stroke reversal also causes vortices to be shed the leading edge sheds a stopping vortex, whereas the dominating vortex for the trailing edge is the starting vortex owing to the rotation. Both these 
vortices are of the 'wrong' sense for the current halfstroke, resulting in a sharp dip in lift (Fig. 9(a)). However, as soon as the subsequent halfstroke commences, these vortices are of the right sense for promoting lift. In addition, a new LEV is also formed, which further increases lift. The rapidity of the stroke reversal ensures that the starting vortex shed from the trailing edge is ejected far away, thereby reducing its inhibitory effect on lift. At the same time, a decreasing angle of attack reduces lift. The overall result is a large increase in lift, seen as a positive spike at the start of each halfstroke (Fig. 9(a)).

The forces on the flapping wing, as generated in the current formulation, are all pressure-related; there is no skin friction because the model is inviscid. Therefore, the thrust component of force is extracted entirely from the same pressure distribution, as is lift. Hence, the similarity between the lift and thrust time histories (c.f. Figs 9(a) and (b)). The main contribution to thrust (or drag, in the conventional sense) comes from the shed wake. As the 'horizontal wake' is substantially greater than the 'vertical wake' (the kinematics being a flat figure-of-eight), thrust is greater than lift (by almost 50 per cent).

Because of the blade-element methodology employed in this work (Part 1, section 3.3), a phase difference exists between wing sections, because the sections travel different distances in the same time depending on their radial position. Consequently, flow in the outboard regions is more developed and 'older' in terms of chord lengths travelled. An important observation made in the current study is that flow behaviour is very strongly linked to the 'age' of the flow in terms of number of chord lengths travelled. It was probably for this reason that Wagner [31] (and later von Kármán and Sears [32]) in his seminal work used semi-chords travelled as the independent variable instead of time. Our observation shows that a reduced-frequency-type parameter is still relevant in this form of unsteady flow.

A consequence of this phase lead-lag is that flow in the outboard sections, having travelled more chord lengths, is more prone to vortex breakdown. A similar observation was made by Ellington et al. $[2,33]$ from experiments on his flapper. Ellington et al. reported that the vortex breakdown caused the LEV to 'lift off' the wing surface in these outboard regions. In such cases, the outermost sections feature a von Kármántype vortex system composed of alternately shedding leading- and trailing-edge vortices. Although this has little effect on mean lift for the wing section, the thrust (drag) component can be significant.

In the current quasi-three-dimensional model, no tip vortex effects are included. Comparison with experimental data, however, has shown good agreement. The reason for this is that vortex breakdown in outboard sections of the wing (and the associated lower lift) produces an effect equivalent to the lower lift that would be expected from tip-vortex effects. A similar argument can be drawn for thrust data. This observation further substantiates the claim made earlier that, at least for the Reynolds numbers in question, three-dimensional effects are subdued and a strip-theory-type extension of the two-dimensional model is capable of reproducing the true three-dimensional characteristics with reasonable accuracy. This was indeed the conclusion drawn by Sane and Dickinson [26] when they compared results from their three-dimensional experiments with Dickinson's earlier two-dimensional experiments [34].

\subsection{Limitations of the model}

Although the model developed in the current study has quite accurately matched experimental observations (Figs 7 and 9), it remains an inviscid, potential, and essentially a two-dimensional model and, therefore, has its limitations.

One such limitation is exposed by the requirement for relaxation of the Kutta-Joukowski condition at the leading and trailing edges, especially during stroke reversals (section 3.2.2.1). In the absence of such a relaxation, the simulations were prone to corruption because of the creation of artificially strong vortices with unrealistically high convection velocities. In reality, it is likely that viscosity (and viscous dissipation) prevents such a situation from arising in the first place, thus avoiding the need to artificially damp the Kutta-Joukowski condition.

Of course, it is also possible that in situations such as insect-like flapping, the Kutta-Joukowski condition takes a somewhat modified form. In the conventional unsteady-flow problems, wing-pitch changes are minimal so that it is reasonable to assume that the smooth-flow condition at the trailing edge applies. During insect-like stroke reversals, however, the pitch manoeuvres are so acute that fluid is more likely to flow around the trailing edge rather than along it. In such cases, the validity of Kutta-Joukowski condition in the conventional sense is, therefore, somewhat dubious. This would provide support for the temporarily relaxed KuttaJoukowski condition used here. More experimental evidence is required, however, before such a claim can be substantiated.

A comparison of Figs 9(a) and (b) shows that the bandwidth of the force-measuring equipment used by Dickinson may have been insufficient in the region of the forces generated. The spikes are always better captured in one direction (negative spikes for lift and positive spikes for thrust) than those in the other direction. Dickinson does not 
report on this characteristic of the experimental setup. It is also possible that the peaks predicted by the theoretical model are, in fact, not observed to the same extent in the experiments because of damping by viscosity and other effects. However, this is unlikely, as this discrepancy occurs in both $\mathcal{L}$ and $\mathcal{T}$ measurements.

Recent work by Leishman and co-workers $[\mathbf{3 5}, \mathbf{3 6}]$ has revealed that tip-vortex effects are important and have a downwash effect on the shed wake. Although such an influence cannot be incorporated in the current modelling framework, an improved model would be needed to resolve this issue. Like Ellington et al. [2], Leishman et al. also noticed a significant spanwise motion of the LEV. The current model does not account for this feature (The spanwise motion of the LEV appears to be dependent on Reynolds number. For small Reynolds numbers ( $R e \sim 200$ ), only weak spanwise flow has been observed $[4,37]$ whereas studies involving higher Reynolds numbers ( $R e \sim 5000$ and higher) have revealed more significant spanwise entrainment $[2,3,35,36]$.) and a method for including it would be useful.

\section{CONCLUSIONS}

In this paper, the analytical equations derived in Part 1 [1] have been converted to forms that are amenable to numerical calculation. This has been necessary, in particular, for the wake integral equations that are exact but do not have a closed analytical form because of their non-linearity.

The problem being solved is the flow associated with insect-like flapping. Such flows are characterized by twin separation, one each from the leading and trailing edges. The shed wakes constitute vortex sheets that move freely at the local Kirchhoff velocity, because they are unable to sustain KuttaJoukowski forces. As a result of insect-like kinematics, the flapping wing repeatedly interacts with its wake because of the back-and-forth motion.

The approach is formulated here as an initial-value problem - using known initial conditions, the flow is solved for all subsequent times using a timemarching algorithm. The calculation relies on vortex methods to implement the solutions. In our approach, the aerofoil is represented by an array of point vortices that are bound but whose strengths are time-varying. The shed wake (both leading and trailing edges) is also represented by an array of discrete point vortices, but these are free and their strengths are fixed by the wake-integral equations at the time of inception.

The method relies on the shed vortices convecting according to the Rott-Birkhoff equation. Convection was executed by means of a simple forward
Euler scheme, and to alleviate the problem of infinite velocities at vortex centres, a desingularization scheme was used. In addition to using LambOseen-type vortices, a vortex amalgamation scheme, whereby vortices of like-sign in close proximity to each other were merged, was also implemented. During the rapid stroke reversals, sub-time-steps were used to increase the resolution of the calculation. It was also found, especially during stroke reversals and wake re-entry, that artificially strong vortices were produced owing to the strong velocity fields, which led to corruption of the solution. In such cases, the Kutta-Joukowski condition was judiciously relaxed for that time-step.

In order to remove, as best as possible, any dependence on numerical parameters, a number of numerical experiments were run. These investigated the effects of quantities such as number of wing sections and time-step-size on the outcome of the simulations. In this way, the best values for the parameters were chosen both in terms of accuracy and practicality. The entire numerical solution was implemented in Fortran. The program size was of the order of $2.0 \mathrm{MB}$ and the memory requirements were determined at runtime, depending on the number of wing sections and time-steps. The bottleneck for the simulations was the convection routine which slowed as the number of vortices increased (varying roughly as $n^{2.5}$, where $n$ is the number of time-steps).

The model was validated both in terms of flow visualization and force prediction. Results from the model showed very good agreement with existing data for low Reynolds number flows. Not only were the vortex structures similar but they were also temporally accurate. Force prediction yielded by the current model was also similarly noteworthy, particularly for thrust data. Comparison of frequency content of this time-dependent flow highlighted the temporal consistency between model and experiment.

These results have shown that the analytical model proposed in Part 1 [1] and implemented here is indeed capable of predicting, with notable accuracy, the forces and flow field generated by an insect-like flapping wing, at least in the range of Reynolds number studied $(R e \approx 200)$. The similarity between their frequency content shows that the underlying flow features are also captured well. In addition, the study has also shown the similarity between two-dimensional and three-dimensional flapping-wing flows at these Reynolds numbers.

\section{ACKNOWLEDGEMENT}

The authors are grateful to the EPSRC (through grant numbers GR/M78472/01 and GR/S23025/01) and the UK Ministry of Defence for supporting this work. 


\section{REFERENCES}

1 Ansari, S. A., Żbikowski, R., and Knowles, K. A nonlinear unsteady aerodynamic model for insect-like flapping wings in the hover. Part 1: methodology and analysis. Proc. IMechE, Part G: J. Aerospace Engineering, 2006, 220(G2), 61-83.

2 Ellington, C. P., van den Berg, C., Willmott, A. P., and Thomas, A. L. R. Leading-edge vortices in insect flight. Nature, 1996, 384, 626-630.

3 Willmott, A. P., Ellington, C. P., and Thomas, A. L. R. Flow visualization and unsteady aerodynamics in the flight of the hawkmoth, Manduca sexta. Phil. Trans. Roy. Soc. Lond. Ser. B, 1997, 352, 303-316.

4 Birch, J. M. and Dickinson, M. H. Spanwise flow and the attachment of the leading-edge vortex on insect wings. Nature, 2001, 412(6848), 729-733.

5 Rosenhead, L. The formation of vortices from a surface of discontinuity. Proc. Roy. Soc. Lond. Ser. A, 1931, 134(823), 170-192.

6 Betz, A. Verhalten von Wirbelsystemen. Zeitschrift für angewandte Mathematik und Mechanik, 1932, 12(3), 164-174.

7 Rott, N. Diffraction of a weak shock with vortex generation. J. Fluid Mech., 1956, 1, 111-128.

8 Birkhoff, G. Helmholtz and Taylor instability. Proceedings of the Symposium on Applied mathematics, 1962, vol. 13, pp. 55-76 (American Mathematical Society, Providence, Rhode Island).

9 Oseen, C. W. Über die Stokes'sche Formel und über eine verwandte Aufgabe in der Hydrodynamik. Arkiv för Matematik, Astronomi och Fysik, 1911, 7(1), 1-36.

10 Lamb, S. H. Hydrodynamics, 6th edition, 1932 (Cambridge University Press, New York).

11 Vatistas, G. H., Kozel, V., and Mih, W. C. A simpler model for concentrated vortices. Exp. Fluids, 1991, 11, 73-76.

12 Spallart, P. R. Vortex methods for separated flows, Technical Memorandum 100068, N88-26342, NASA, 1988.

13 Sarpkaya, T. Computational methods with vortices the 1988 Freeman Scholar Lecture. Trans. ASME, J. Fluids Eng., 1989, 111, 5-52.

14 Ting, L. and Klein, R. Viscous vortical flows, lecture notes in physics, 1991, vol. 374 (Springer-Verlag, Berlin).

15 Ansari, S. A. A nonlinear, unsteady, aerodynamic model for insect-like flapping wings in the hover with micro air vehicle applications. $\mathrm{PhD}$ Thesis, Cranfield University (RMCS Shrivenham), September 2004.

16 Katz, J. and Plotkin, A. Low-Speed aerodynamics, 2nd edition, 2001 (Cambridge Univeristy Press, Cambridge).

17 Crighton, D. G. The Kutta condition in unsteady flow. Ann. Rev. Fluid Mech., 1985, 17, 411-445.

18 Silverstein, A. and Joyner, U. T. Experimental verification of the theory of oscillating airfoils, Report 673, NACA, 1939.

19 Leishman, J. G. Principles of helicopter aerodynamics, 2000 (Cambridge University Press, Cambridge).

20 Giesing, J. P. Vorticity and Kutta condition for unsteady multienergy flows. Trans. ASME, J. Appl. Mech., 1969, 36, 608-613.

21 Maskell, E. C. On the Kutta-Joukowski condition in two-dimensional unsteady flow. TM ARC-33967, Royal Aircraft Establishment, Farnborough, England, 1972.
22 Mook, D. T. and Dong, B. Perspective: numerical simulations of wakes and blade-vortex interaction. Trans. ASME, J. Fluids Eng., 1994, 116(1), 5-21.

23 Poling, D. R. and Telionis, D. P. The response of airfoils to periodic disturbances - the unsteady Kutta condition. AIAA J., 1986, 24(2), 193-199.

24 Poling, D. R. and Telionis, D. P. The trailing edge of a pitching airfoil at high reduced frequencies. Trans. ASME, J. Fluids Eng., 1987, 109, 410-414.

25 Sun, Q. and Boyd, I. D. Flat-plate aerodynamics at very low Reynolds number. J. Fluid Mech., 2004, 502, 199-206.

26 Sane, S. P. and Dickinson, M. H. The aerodynamic effects of wing rotation and a revised quasi-steady model of flapping flight. J. Exp. Biol., 2002, 205, 1087-1096.

27 Dickinson, M. H. and Götz, K. G. Unsteady aerodynamic performance of model wings at low reynolds numbers. J. Exp. Biol., 1993, 174, 45-64.

28 Sun, M. and Tang, J. Unsteady aerodynamic force generation by a model fruit fly wing in flapping motion. J. Exp. Biol., 2002, 205, 55-70.

29 Dickinson, M. H., Lehmann, F.-O., and Sane, S. P. Wing rotation and the aerodynamic basis of insect flight. Science, 1999, 284, 1954-1960.

30 Ansari, S. A., Żbikowski, R., and Knowles, K. A nonlinear unsteady aerodynamic model for insect-like flapping wings in the hover. Part 2: implementation and validation. Proc. IMechE, Part G: J. Aerospace Engineering, 2006, 220(G3), 169-186.

31 Wagner, H. Über die Entstehung des Dynamischen Aufftriebes von Tragflügeln. Zeitschrift für Angewandie Mathematik und Mechanik, 1925, 5(1), 17-35.

32 von Kármán, T. and Sears, W. R. Airfoil theory for non-uniform motion. J. Aeronaut. Sci., 1938, 5(10), 379-390.

33 van den Berg, C. and Ellington, C. P. The three-dimensional leading-edge vortex of a 'hovering' model hawkmoth. Phil. Trans. Roy. Soc. Lond. Ser. B, 1997, 352(1351), 329-340.

34 Dickinson, M. H. The effects of wing rotation on unsteady aerodynamic performance at low Reynolds numbers. J. Exp. Biol., 1994, 192, 179-206.

35 Ramasamy, M., Leishman, J. G., and Singh, B. Wake structure diagnostics of a flapping wing MAV. In SAE International Powered Lift Conference, Texas, 3-6 October 2005, IPLC 2005-01-3198, pp. 1-13.

36 Tarascio, M. J., Ramasamy, M., Chopra, I., and Leishman, J. G. Flow visualization of micro air vehicle scaled insect-based flapping wings. J. Aircraft, 2005, 42(2), 385-390.

37 Sane, S. P. and Dickinson, M. H. The control and flight force by a flapping wing: lift and drag production. $J$. Exp. Biol., 2001, 204, 2607-2626.

\section{APPENDIX}

\section{Notation}

$\mathcal{D} \quad$ horizontal drag force orthogonal to gravity (N)

$h \quad$ heave displacement (m) 
$\dot{h}$ heave velocity $(\mathrm{m} / \mathrm{s})$

$l \quad$ lunge displacement (m)

$\dot{l} \quad$ lunge velocity $(\mathrm{m} / \mathrm{s})$

$\mathcal{L} \quad$ vertically upwards lift force parallel to gravity $(\mathrm{N})$

$\mathcal{M}$ moment about wing pitch axis (Nm)

$q$ complex velocity $(\mathrm{m} / \mathrm{s})$

$r \quad$ radial distance from centre $(\mathrm{m})$

$r_{c} \quad$ vortex-core radius $(\mathrm{m})$

$R \quad$ radius of circle in $\mathcal{Z}$-plane (m)

$R$ real part of

$t \quad$ time (s)

$\mathcal{T}$ horizontal thrust force, parallel to drag, but always in the direction of motion $(\mathrm{N})$

$U_{\mathrm{E}} \quad$ horizontal $(\tilde{\xi})$ component of external irrotational velocity field $(\mathrm{m} / \mathrm{s})$

$V_{\mathrm{E}} \quad \operatorname{vertical}(\tilde{\eta})$ component of external irrotational velocity field $(\mathrm{m} / \mathrm{s})$

$v_{\theta} \quad$ circumferential component of velocity $(\mathrm{m} / \mathrm{s})$

$x \quad$ real-axis coordinate in circle $(\mathcal{Z})$ plane $(\mathrm{m})$

$y \quad$ imaginary-axis coordinate in circle $(\mathcal{Z})$

plane $(\mathrm{m})$

$\mathcal{Z} \quad$ complex coordinates in circle plane $(=x+\imath y)(\mathrm{m})$

$\breve{A} \quad$ rotating coordinate system

$\hat{A} \quad$ translating coordinate system

$\tilde{A} \quad$ inertial coordinate system
$\bar{A} \quad$ complex conjugate of

$\dot{A} \quad$ first derivative w.r.t. time $\left(\mathrm{s}^{-1}\right)$

$\alpha \quad$ angle of attack (rad)

$\dot{\alpha} \quad$ pitch rate $(\mathrm{rad} / \mathrm{s})$

$\gamma \quad$ vorticity $(\mathrm{m} / \mathrm{s})$

$\delta \quad$ elemental value of

$\Gamma \quad$ circulation $\left(\mathrm{m}^{2} / \mathrm{s}\right)$

$\epsilon \quad$ amalgamation threshold $\left(\mathrm{m}^{3} / \mathrm{s}\right)$

$\zeta \quad$ complex coordinates in physical plane $(=\xi+\imath \eta)(\mathrm{m})$

$\eta \quad$ imaginary-axis coordinate in physical $(\zeta)$ plane (m)

$\theta \quad$ inclination angle (rad)

$\theta \quad$ angle along circle in $\mathcal{Z}$-plane

$\Theta \quad$ inclination amplitude (rad)

$l \quad$ complex $\imath(=\sqrt{ }-1)$

$\xi \quad$ real-axis coordinate in physical ( $\zeta)$ plane $(\mathrm{m})$

$\rho \quad$ fluid density $\left(\mathrm{kg} / \mathrm{m}^{3}\right)$

$\phi \quad$ stroke angle (rad)

$\Phi \quad$ stroke amplitude (rad)

\section{Subscripts}

$A_{0} \quad$ wake-free (quasi-steady) component

$A_{1} \quad$ wake-induced component

$A_{\mathrm{lv}} \quad$ leading-edge vortex

$A_{\mathrm{wk}} \quad$ trailing-edge wake 